\title{
Radial consolidation of a phase-change soil
}

\author{
R. F. SCOTT*
}

An overconsolidated sensitive clay has been represented by a one-dimensional compressional model in which the clay structure is rigid both below and above the maximum past effective stress and collapses at this stress from a higher void ratio to a lower value. This model is incorporated in several different geometries to describe the behaviour of the sensitive clay under applied stress. These cases include radial compression of a cylinder and a sphere, and expansion of a cylindrical cavity in an infinite region. Solutions are given for displacements and pore pressures.

KEYWORDS: analysis; consolidation; phase change; radial; sensitive clay; spherical
Une argile sensible surconsolidée est représentée par un modèle unidimensionnel de compression dans lequel la structure de l'argile est rigide à des valeurs aussi bien supérieures qu'inférieures à la valeur maximale passee de la contrainte effective et s'effondre au niveau de celle-ci en acquérant un indice des vides plus faible. Le modèle est incorporé dans plusieurs géometries différentes pour décrire le comportement de l'argile sensible sous contrainte appliquée. Ces cas comprennent la compression radiale d'un cylindre et d'une sphère et l'expansion d'une cavité cylindrique dans un milieu infini. Des solutions sont présentées pour les déplacements et pour les pressions interstitielles.

\section{INTRODUCTION}

Overconsolidated sensitive clays exhibit a highly non-linear consolidation behaviour. Their response to applied load has generally been represented by an incremental numerical approach in which the compressibility and permeability of the clay have been varied, following the laboratory test behaviour, during the consolidation process (Mesri \& Rokhsar, 1983; Mesri \& Choi, 1985). Consolidation and settlement calculations, described this way, have required the use of a computer. Recently, for one-dimensional compression, the laboratory behaviour of such soils has been represented differently, by assuming at the simplest level that the clay under applied stress exhibits no compression up to the preconsolidation pressure, at which it collapses to a new void ratio where it remains, again rigid, for higher pressures (Scott, 1989). The process is shown in Fig. 1, which illustrates the typical behaviour of such a clay in laboratory tests and the idealization of the model to represent such behaviour; an arithmetic scale of stress is given in both plots. In effect, under a stress increment $\Delta \sigma_{v}^{\prime}$ from effective stress $\sigma_{0}^{\prime}$ to stress $\sigma_{\mathrm{f}}^{\prime}$, the clay undergoes a phase change at the maximum past effective stress $\sigma_{\mathrm{p}}^{\prime}$ from an initial void ratio $e_{1}$ to a final state at

Discussion on this Paper closes on 1 October 1990. For further details, see p. ii.

* California Institute of Technology, Pasadena. lower void ratio, $e_{2}$. The letters A, B etc. on Fig. 1(a) correspond to material states at points $A_{1}$, $B_{1}$ etc. on Fig. 1(b). The possibility of a more complicated model (Scott, 1989) is allowed for by establishing an intermediate point $\mathrm{C}$. The more complicated model admits the possibility of studying the application of the phase-change process to cylindrical or spherical geometries to examine the pore pressure in the central core by analogy with the Mandel-Cryer effect (Cryer, 1963). This is not treated here.

When a surface load is applied to a half-space composed of such a clay, with drainage at the surface, the soil collapses to the lower void ratio first at the surface, and this collapse releases pore water at the collapse front, which is a shock wave passing into the undisturbed medium. The flow of the released water through the increasing thickness of the collapsed zone to the drainage surface controls the rate of advance of the shock in the simplest one-dimensional case of uniform homogeneous soil and a step load at the ground surface according to the equation

$$
\Delta v \frac{\mathrm{d} Z}{\mathrm{~d} t}-\frac{k_{\mathrm{s}} u_{\mathrm{p}}}{\gamma_{\mathrm{w}}\left(Z-z_{0}\right)}=0
$$

in which $\Delta v$ is the volume of water expelled by unit volume of the original sensitive clay in the phase change from $e_{1}$ to $e_{2}$ (i.e., $\Delta v=\left(e_{1}-e_{2}\right) /(1$ $\left.+e_{1}\right)$ ), $Z$ is the distance from the original surface 


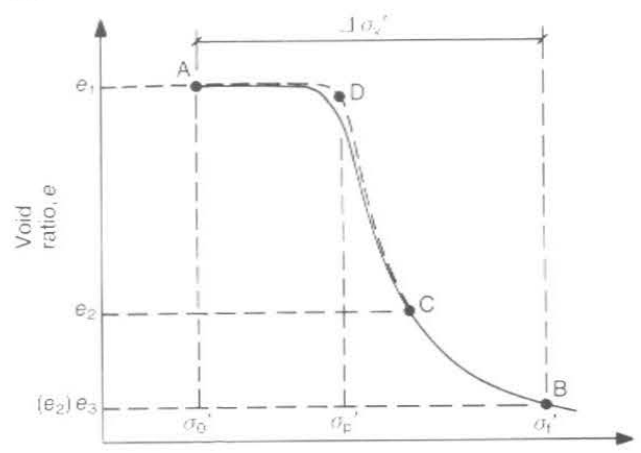

(a)

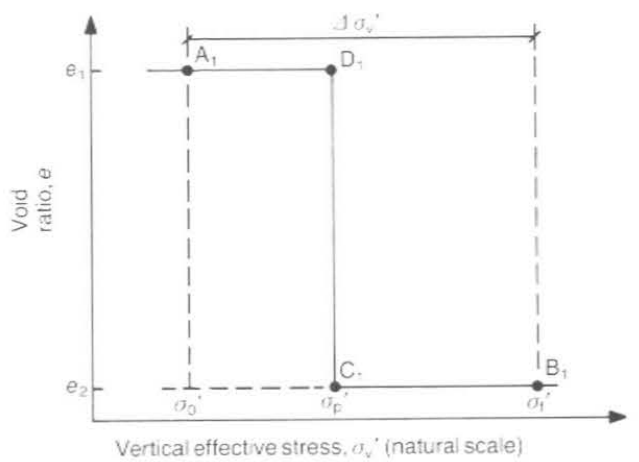

(b)

Fig. 1. Behaviour of overconsolidated sensitive clay in one-dimensional consolidation test (note linear scale of stress): (a) laboratory test; (b) idealization

(reference level) to the advancing front, $t$ is time, $k_{\mathrm{s}}$ is the permeability of the collapsed soil, $u_{\mathrm{p}}$ is the excess pore pressure at $Z, \gamma_{w}$ is the unit weight of water, and $z_{0}$ is the settlement of the soil surface (not assumed small) from the original surface reference. The settlement $z_{0}$ is obviously related to the thickness of the collapsed layer by the equation

$$
z_{0}=\Delta v Z
$$

To generate the process it is necessary that the applied pressure increment increase the effective stress in the soil from its existing value (see Fig. 1) to a value in excess of the maximum past consolidation pressure; otherwise no drainage or settlement will occur. When the maximum past pressure is exceeded, the pore pressure $u_{\mathrm{p}}$ generated at the shock front is equal to the difference between the total applied stress and the maximum past pressure (Fig. 1). For uniform soil with constant maximum past pressure with depth, and a step application of load, thereafter constant, the integration of a rearranged equation (1) gives the simple result that $Z$ (and therefore $z_{0}$ ) varies with the square root of time

$$
Z=2 a t^{1 / 2}
$$

where

$$
a^{2}=\frac{k_{\mathrm{s}} u_{\mathrm{p}}}{2 \gamma_{\mathrm{w}}(1-\Delta v) \Delta v}
$$

In a previous paper (Scott, 1989), this result was extended to a case of a layer of sensitive clay, and calculations of settlement were found to compare well with measured values at the Olga B test site (Mesri \& Choi, 1985). Here the analysis is extended to treat cases of radial consolidation, when the driving stress increment is applied first to the outside of an infinite cylinder of sensitive clay behaving according to Fig. 1, and second to the inside surface of an infinitely long cylindrical hole in such a clay.

\section{PRESSURE APPLIED TO OUTSIDE OF} INFINITE SOLID CIRCULAR CYLINDER

The first situation is shown in Fig. 2, at a time $t$ after the step application of a stress increment $\Delta \sigma^{\prime}$ to the outside of a cylinder of overconsolidated sensitive clay. As in Fig. 1, this applied stress is sufficient to generate a constant pressure $u_{\mathrm{p}}$ in the pore water at the advancing shock front, where $u_{\mathrm{p}}$ is the difference between the applied stress and the maximum past effective stress. Here the position of the shock front is described by the radius $R$, which decreases with time. The original radius of the cylinder is $R_{0}$, and the radius of the compressed cylinder is $r_{0}$. The controlling equation again comes from the rate of flow condition, where the flow generated by the advancing shock is conducted through the permeable collapsed layer between $R$ and $r_{0}$ to the outer drainage surface at $r_{0}$. The difference from the plane problem is due to the radial geometry, and the fact that $R$ is shrinking. The equation is

$$
-\Delta v \frac{\mathrm{d} R}{\mathrm{~d} t} 2 \pi R-\text { rate of flow from } R \text { to } r_{0}=0
$$

But the flow condition is one of onedimensional radial flow, so that the rate $q$ in the annulus between $R$ and $r_{0}$ is (Scott, 1963)

$$
q=\frac{2 \pi k_{\mathrm{s}} u_{\mathrm{p}}}{\gamma_{\mathrm{w}} \ln r_{0} / R}
$$

and, substituting in equation (5), the control equation becomes 


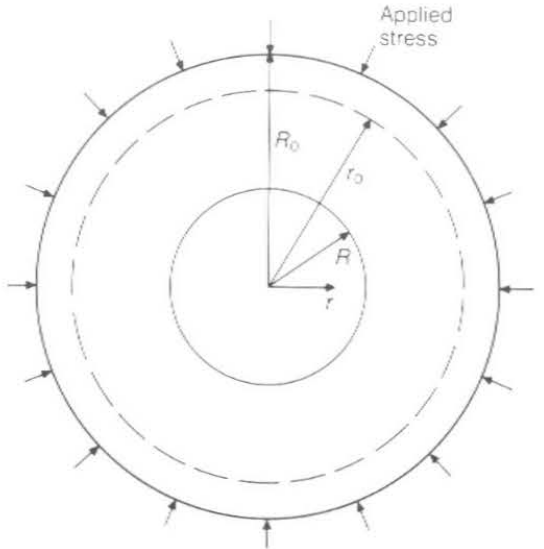

Fig. 2. Phase change in infinite solid circular cylinder under pressure applied at outside boundary

$$
-\Delta v \frac{\mathrm{d} R}{\mathrm{~d} t} R-\frac{k_{\mathrm{s}} u_{\mathrm{p}}}{\gamma_{\mathrm{w}} \ln r_{0} / R}=0
$$

The relationship of $r_{0}$ to $R$ is also different from the plane case because of the radial symmetry. At the time $t$, shown in Fig. 2, all the soil at void rato $e_{1}$ formerly occupying the annulus or volume between $R_{0}$ and $R$ now has been compressed to void ratio $e_{2}$ in the volume between $R$ and $r_{0}$. Consideration of the volumes and void ratios gives the equation for the ratio of new volume to original volume

$$
\frac{r_{0}^{2}-R^{2}}{R_{0}^{2}-R^{2}}=\frac{1+e_{2}}{1+e_{1}}=b, \text { say }
$$

where $b$ is a new constant lying between the limits zero and unity. It can be seen that this new constant is related to $\Delta v$ by the relation $\Delta v=1-b$. At this stage, it is convenient to define dimensionless terms

$$
R^{\prime}=R / R_{0} ; \quad r_{0}^{\prime}=r_{0} / R_{0}
$$

where $0<R^{\prime}<1 ; b<r_{0}^{\prime}<1$, so that, with some rearranging, equation (7) becomes

$$
-\left(\ln r_{0}^{\prime} / R^{\prime}\right) R^{\prime} \mathrm{d} R^{\prime}=\frac{k_{\mathrm{s}} u_{\mathrm{p}}}{R_{0}^{2} \Delta v \gamma_{\mathrm{w}}} \mathrm{d} t
$$

Both sides are now dimensionless and it can be seen that a group of terms on the right-hand side has the dimensions of a diffusion coefficient, or coefficient of consolidation, say $c_{\mathrm{vp}}$, for this problem

$$
c_{\mathrm{vp}}=\frac{k_{\mathrm{s}} u_{\mathrm{p}}}{\gamma_{\mathrm{w}}}
$$

Substituting equation (8) in equation (10) and rearranging gives the equation to be solved, in which the prime on $R^{\prime}$ has been dropped

$$
\begin{gathered}
-R \ln \left\{\frac{\left[R^{2}(1-b)+b\right]^{1 / 2}}{R}\right\} \mathrm{d} R \\
=\frac{k_{\mathrm{s}} u_{\mathrm{p}}}{R_{0}{ }^{2} \gamma_{\mathrm{w}}(1-b)} \mathrm{d} t
\end{gathered}
$$

The solution is obtained by integration of the left side from $R=1$ to $R$, and the right side from $t=0$ to $t$, to give

$$
\begin{aligned}
& \frac{(1-b)}{4}\left\{2 R^{2} \ln R-\left(R^{2}+\frac{b}{1-b}\right)\right. \\
& \left.\times \ln \left[(1-b) R^{2}+b\right]\right\}=\frac{k_{\mathrm{s}} u_{\mathrm{p}} t}{R_{0}^{2} \gamma_{\mathrm{w}}}=T
\end{aligned}
$$

where $T$ is a dimensionless time, defined as

$$
T=\frac{k_{\mathrm{s}} u_{\mathrm{p}} t}{\gamma_{\mathrm{w}} R_{0}^{2}}=\frac{c_{\mathrm{vp}} t}{R_{0}^{2}}
$$

The phase change process terminates at a time $t_{\mathrm{f}}$ when the entire cylinder has collapsed to the new state at void ratio $e_{2}$, with $R=0$. Placing $R=0$ in equation (13) gives

$$
t_{\mathrm{f}}=\frac{R_{0}^{2} \gamma_{\mathrm{w}} b \ln 1 / b}{4 k_{\mathrm{s}} u_{\mathrm{p}}}
$$

or

$$
T_{\mathrm{f}}=\frac{b \ln 1 / b}{4}
$$

It may be noted here that, although of less practical use, the one-dimensional compressional problem of spherical symmetry can be treated similarly. In the spherical case the solution is

$$
\begin{aligned}
& \frac{(1-b)}{2}\left[\left(1-R^{2}\right)-\frac{1}{(1-b)}\right. \\
& \left.\quad \times\left\{1-\left[(1-b) R^{3}+b\right]^{2 / 3}\right\}\right]=\frac{u_{\mathrm{p}} k_{\mathrm{s}} t}{{R_{0}}^{2} \gamma_{\mathrm{w}}}=T
\end{aligned}
$$

in which all the variables and constants have the same meaning as in the radial problem, but refer to the spherical case. Once again, $t=t_{\mathrm{f}}$ at the end of the process, when $R=0$, and, from equation (16)

$$
t_{\mathrm{f}}=\frac{R_{0}{ }^{2} \gamma_{\mathrm{w}}\left(b^{2 / 3}-b\right)}{2 k_{\mathrm{s}} u_{\mathrm{p}}}
$$


or

form

$$
T_{\mathrm{f}}=\frac{b^{2 / 3}-b}{2}
$$

The solutions in terms of the position of the advancing shock front for the cylindrical and spherical cases (equations (13) and (16)) are compared in Fig. 3 for five values of the constant $b$, in terms of dimensionless time $T$, and the square root of dimensionless time.

In Figs 3(b) and (d), the behaviour of the advancing front is seen to correspond to a linear variation with the square root of dimensionless time at small times in all cases. If, in equation (3), a dimensionless distance $Z^{\prime}$ is defined, where $Z^{\prime}=Z / H$, then equation (3) can be recast in the

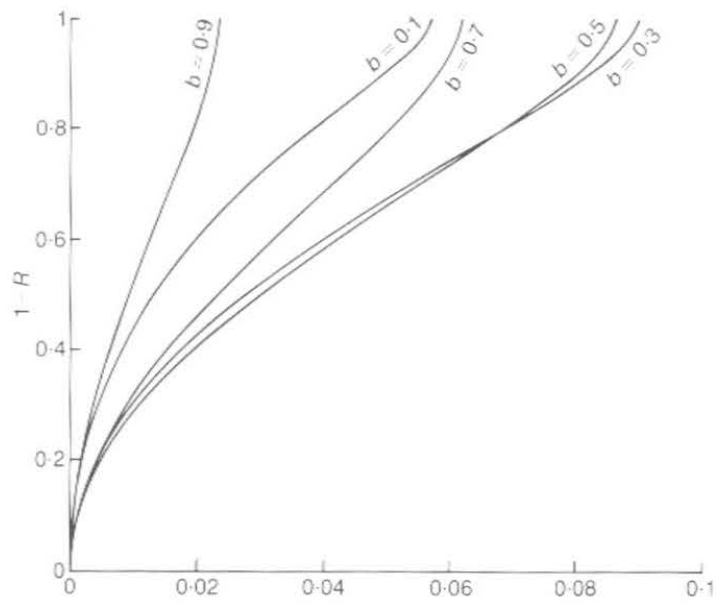

(a)

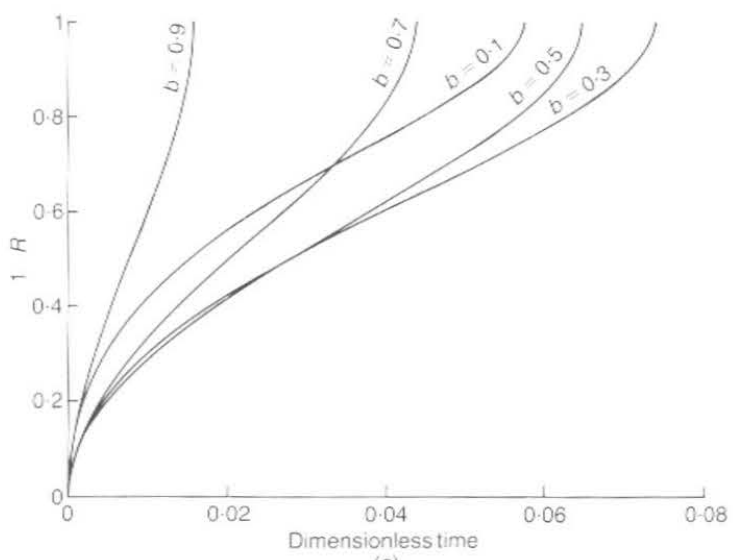

(c)

$$
Z^{\prime}=\left\{\frac{2 T}{b(1-b)}\right\}^{1 / 2}
$$

if $T$ is defined as in equation (14) with $H$ replacing $R_{0}$. This is a straight line of slope $[2 /$ $b(1-b)]^{1 / 2}$ on a plot of $Z^{\prime}$ against $T^{1 / 2}$. It is seen that the slope is the same for pairs of values of $b$ and $1-b$, as illustrated in Fig. 3 for both radial and spherical solutions which can be shown to be asymptotic to equation (18) at small values of $T$. At larger times both radial and spherical solutions diverge from the one-dimensional slab case to approach the appropriate final values of $T_{\mathrm{f}}$ more rapidly, because of the influence of the effectively two- and three-dimensional drainage paths.

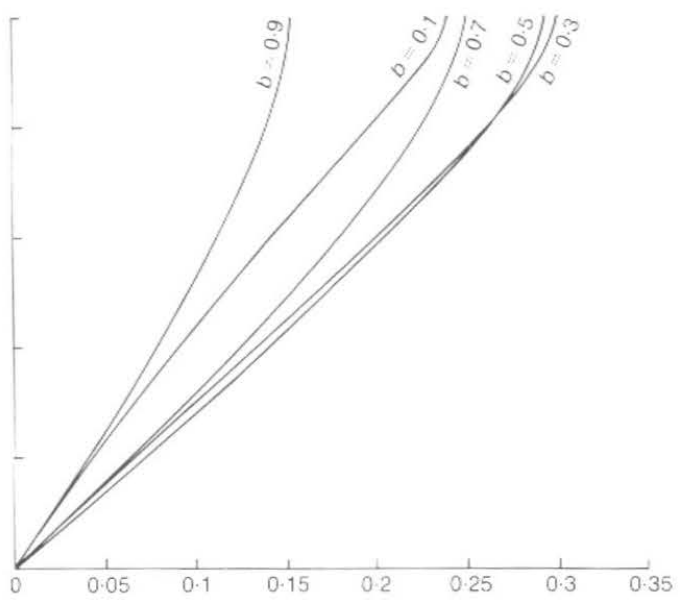

(b)

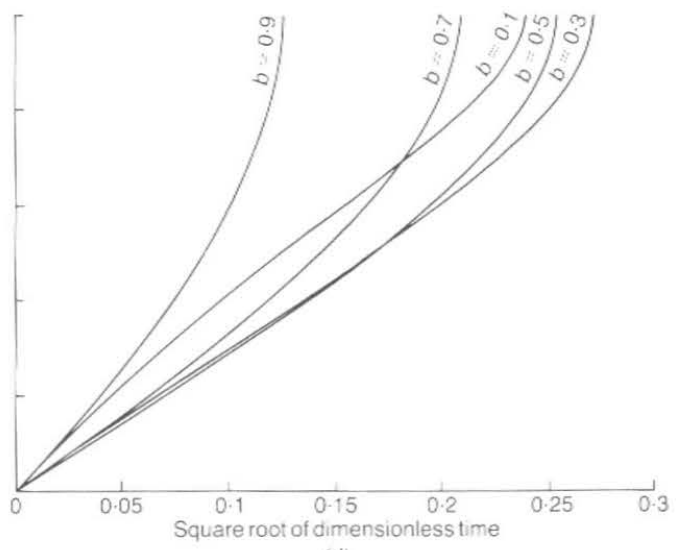

(d)

Fig. 3. Solution for displacement of surface as a function of dimensionless time: (a) cylindrical case, plot of $1-R$ against $T$; (b) cylindrical case, plot of $1-R$ against $T^{1 / 2}$; (c) spherical case, plot of $1-R$ against $T$; (d) spherical case, plot of $1-R$ against $T^{1 / 2}$ 


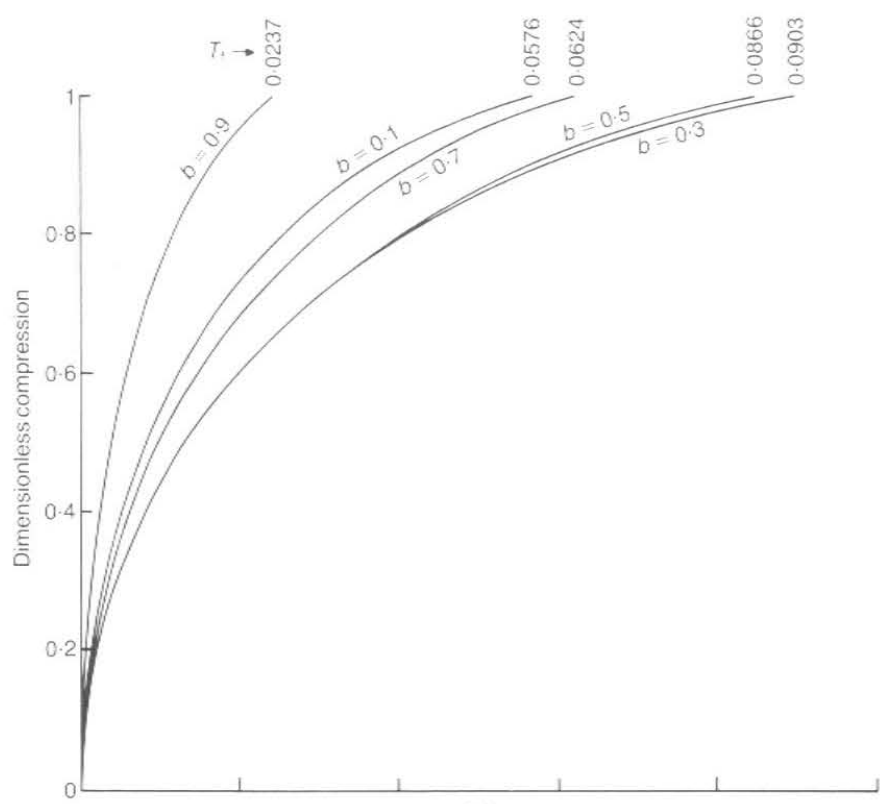

(a)

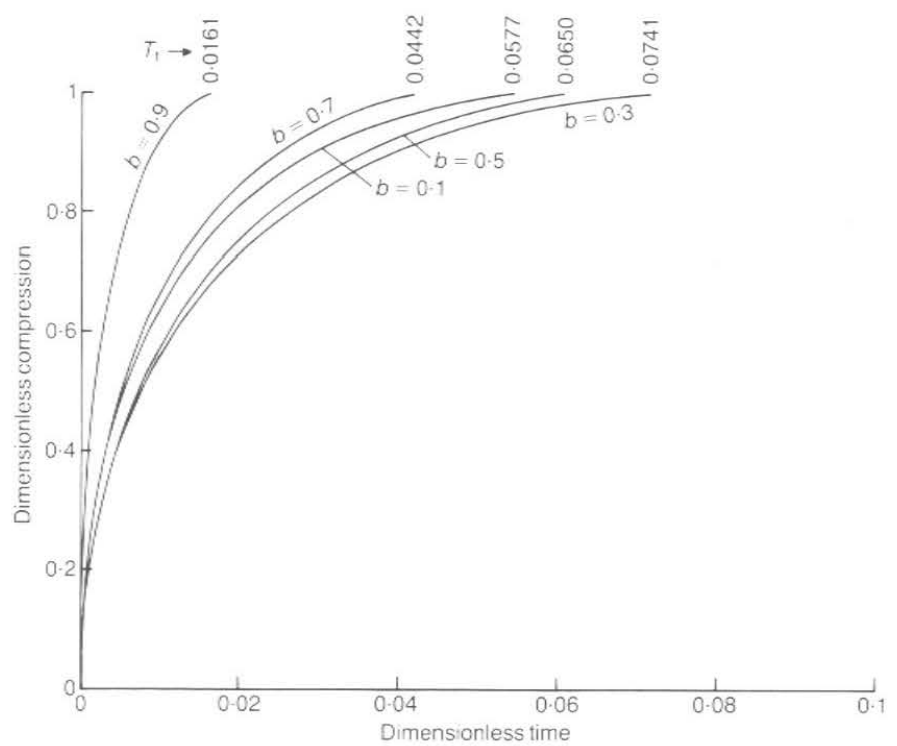

(b)

Fig. 4. Dimensionless compression versus dimensionless time: (a) cylindrical case; (b) spherical case

An interesting aspect of the solutions shown in Fig. 3 is that the time to complete consolidation $(R=0)$ reaches a maximum, and then diminishes as $b$ decreases. It might be expected that, for soil which collapsed more for the same pressure increment (decreasing $b$ ), the necessity for removing increasing quantities of water at the shock interface would result in monotonically increasing consolidation times. However, at the same time, $r_{0}$ is also decreasing, which cuts down the distance that the released water has to travel to the drainage surface. Eventually the effect of waning radius overcomes the volume of water to be drained, and the time decreases for small (and perhaps, at least for soils, unrealistic) values of $b$. By differentiation of equations (15b) and (17b) for 


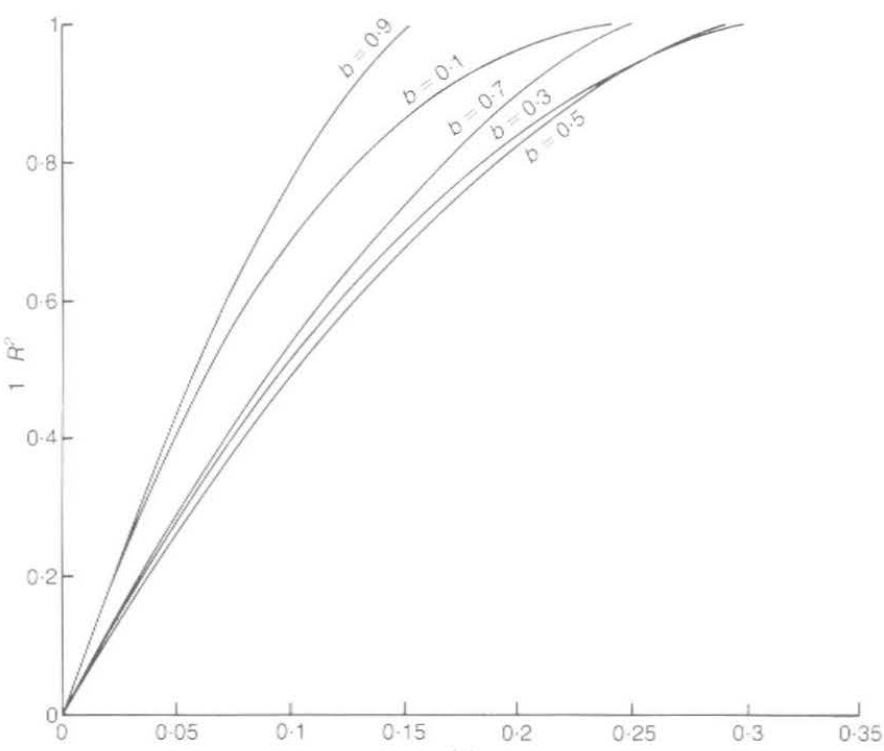

(a)

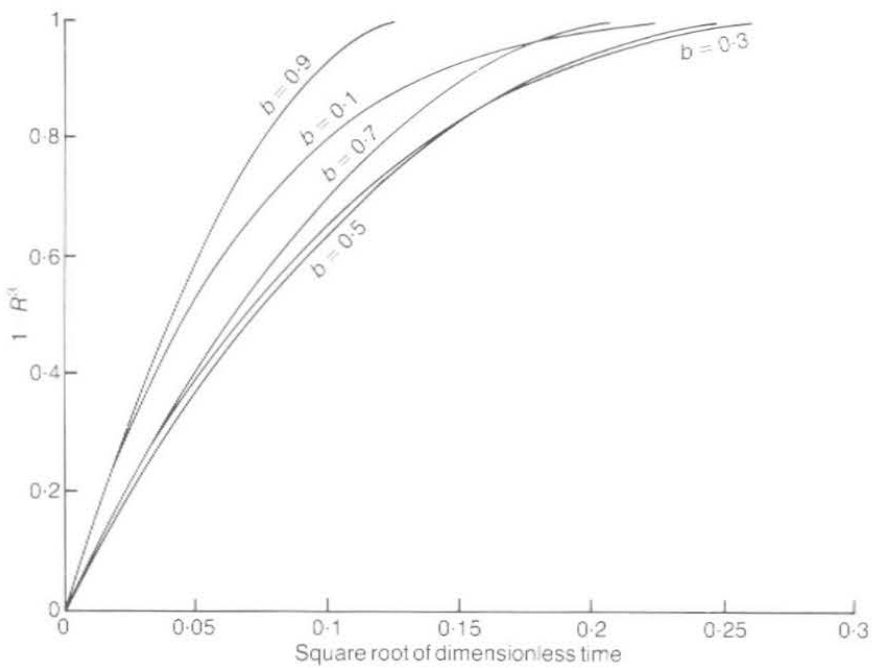

(b)

Fig. 5. Degree of consolidation on a volumetric basis as a function of dimensionless time: (a) cylindrical case, plot of $1-R^{2}$ against $T^{1 / 2}$; (b) spherical case, plot of $1-R^{3}$ against $T^{1 / 3}$

cylindrical and spherical cases, respectively, the maximum value $T_{\mathrm{fm}}$ is $1 / 4 e=0.0920$ for $\mathrm{a} b$ of $1 / e=0.3679$ for the cylinder, and $T_{\mathrm{fm}}$ is $2 /$ $27=0.0741$ for $b=(2 / 3)^{3}=0.2963$ for the sphere. Values of $T_{\mathrm{f}}$ for the various values of $b$ are shown in Fig. 4.

In the plane one-dimensional case the curve shown also represents $U_{z}$, the degree of consolidation, since the settlement, $z_{0}$, is just equal to the distance $Z$ times the constant factor $\Delta v$. However, in the radial and spherical problems, the relationship of the contraction radius, $r_{0}$, to $R$ is more complicated, being represented by equation (8) in the radial case and by the equation

$$
\frac{r_{0}{ }^{3}-R^{3}}{1-R^{3}}=b
$$

in the spherical case, where $r_{0}$ and $R$ are both in their dimensionless forms. In these circumstances, the degree of consolidation, expressed in terms of 
the contraction of the cylindrical or spherical element, is given by the equation

$$
U_{\mathrm{r}}(\text { radial }) \text { or } U_{\mathrm{s}}(\text { spherical })=\frac{1-r_{0}}{1-r_{0 f}}
$$

In equation (20), $r_{0}$ is obtained from the dimensionless form of equation (8)

$$
\frac{r_{0}^{2}-R^{2}}{1-R^{2}}=b
$$

for cylindrical consolidation, and from equation (19) for spherical consolidation. Since at the completion of consolidation $R=0$ in both cases, $r_{\text {of }}$ can be obtained by substitution of $R=0$ in equations (21) and (19) to give respective values of $r_{\text {of }}$ of $b^{1 / 2}$ and $b^{1 / 3}$ for the radial and spherical cases. Combining equation (20) with equation (21) or equation (19) gives expressions for the degree of consolidation in terms of displacement, referred to here as 'dimensionless compression' for the radial and spherical problems, respectively. For different values of $b$, the dimensionless compressions for the two cases are plotted against time in Fig. 4, in which the terminal values of dimensionless time, $T_{\mathrm{f}}$, are also illustrated.

A third possibility for degree of consolidation in problems of radial and spherical symmetry is to define it by reference to the ratio of the volume of settlement, or volume of expressed fluid to the final volume. With this approach, degree of consolidation becomes

$$
U_{\mathrm{rv}}=\frac{1-r_{0}^{2}}{1-r_{\mathrm{Of}}^{2}}
$$

for radial compression, and

$$
U_{\mathrm{sv}}=\frac{1-r_{0}{ }^{3}}{1-r_{\mathrm{of}}{ }^{3}}
$$

in the spherical case. Both of these expressions, along with equations (8) and (19), lead to simpler equations linking $U$ and $R$

$$
U_{\mathrm{rv}}=1-R^{2}
$$

and

$$
U_{\mathrm{sv}}=1-R^{3}
$$

The one-dimensional plane representation of $U$, remains unchanged, of course, with these different definitions. In Fig. 5, $U_{\mathrm{rv}}$ and $U_{\mathrm{sv}}$ from equations (24) and (25) are shown plotted against the square root of dimensionless time to give better definition of the consolidation values at small times.
Since the cylindrical and spherical problems correspond to the geometry and boundary conditions of the Mandel-Cryer effect (de Leeuw, 1964; Cryer, 1963), the pore pressure at the centre $(r=0)$ is of interest. The history is simple: in each case, the central pore pressure rises immediately to the value $u_{\mathrm{p}}$, at which it stays constant until the time $T_{\mathrm{f}}$, where it drops abruptly to zero as the phase change reaches the centre.

\section{PRESSURE APPLIED TO INSIDE OF INFINITE CYLINDRICAL HOLE}

From a field testing point of view, the condition where a pressure is applied to the inside of a cavity in an infinite homogeneous clay medium is also of interest. This problem is more complicated than the case of consolidation of a finite solid clay cylinder stressed externally.

In a mass of uniform, homogeneous, sensitive overconsolidated clay the lateral effective stress at any depth may be less than, equal to, or greater than the present overburden effective stress, depending on the maximum past vertical effective stress, the present vertical stress and the depth. The maximum past lateral effective stress will be less than the maximum past vertical stress, but the present lateral effective stress will still be less than the maximum past stress. If a vertical circular cylindrical hole is drilled into the soil, the lateral stress on the hole wall may be reduced to zero, or maintained at some stress if drilling mud is used. It is assumed for the following analysis that a cylindrical cavity expansion apparatus is lowered into the borehole, and that the equipment has a length several times its diameter, so that the pressurized part of the hole can be considered effectively infinite in length. Further, the apparatus is special in that a drainage layer is interspersed between the pressurizing tube and the hole wall, so that consolidation of the clay can take place by drainage into this layer. As far as is known, such a device is not at present available. It will be assumed in the following analysis that the water pressure at the test depth in the hole has the same value as the hydrostatic pressure in the undisturbed clay.

The apparatus is expanded to apply a known pressure to the inner surface of the hole; the pressure is greater than the maximum past lateral effective stress, so that the clay will begin to collapse at the inner boundary of the hole as a result of drainage of pore water to the inner boundary. As in previous cases, a cylindrical phase change boundary or shock will begin at the inner hole surface and propagate outwards, leaving a growing annulus of collapsed soil behind it, and 
this will result in an increasing radius of the hole itself, so that the distension apparatus, as usual, must be capable of following the hole surface, while maintaining a constant pressure on it. The principal difference in this case from previous ones is that the lateral stress at the radius of the shock decreases as the radius increases, even with constant lateral stress at the hole wall. Eventually, the shock will reach a radius at which the lateral stress has dropped down to the maximum past lateral effective stress, and the phase change process will stop, with the expansion of the cavity wall reaching a limit value.

In this geometry the compressional radial stress is accompanied by an extensional tangential stress, but it is assumed here for a first analysis of the problem that the phase change is dependent only on the compressive radial stress, as in the plane one-dimensional case. Since the clay is assumed to be rigid both before and after collapse, the variation of the radial compressive stress with radius is obtained by consideration of radial equilibrium, and therefore diminishes with the square of the radius. The geometry of the problem is shown at times $t$ in Fig. 6, where part (a) shows a horizontal section through the hole,
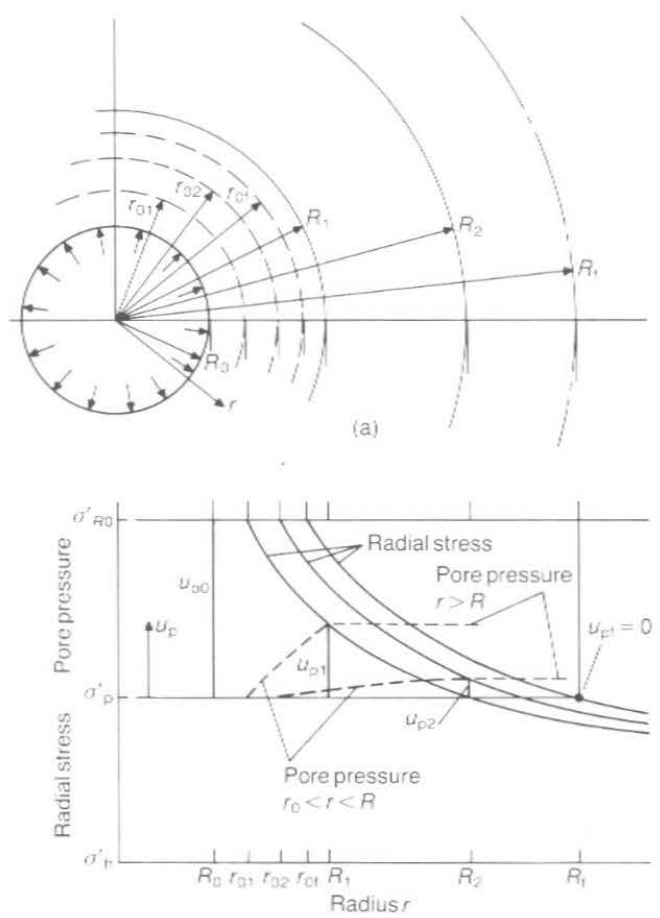

(b)

Fig. 6. Phase change in infinite solid due to pressure applied at internal circular boundary: (a) plan view showing geometry with shock boundary: (b) elevation showing pressure distribution at various times of initial radius $R_{0}$, with $R$ the radius of the shock, and $r_{0}$ the radius of the expanded cavity at $t$. Fig. 6(b) shows the radial stress distributions at various times and the pore pressure variation as a function of radius between $r_{0}$ and $R$.

At the phase boundary, the excess over hydrostatic pore pressure, $u_{\mathrm{p}}$, driving water through the collapsed annulus to the inner hole boundary at $r_{0}$ is the difference between the effective radial pressure, $\sigma_{R}^{\prime}$, generated at the shock and the maximum past lateral effective stress, $\sigma_{\mathrm{p}}^{\prime}$, in the clay. At great radial distances from the hole the lateral effective stress is the static value $\sigma_{\mathrm{h}}^{\prime}$. It is noted that, since the soil is assumed to be rigid everywhere, at the borehole wall the total radial stress applied does not generate any change in the static pore water pressure. Thus the total radial stress and the effective radial stress both decay inversely as the square of radius. At the borehole wall, radius $R_{0}$, the applied radial effective stress is $\sigma_{R v}^{\prime}$. At any radius, $r$, the radial effective stress is given by the equation

$$
\sigma_{\mathrm{r}}^{\prime}-\sigma_{\mathrm{h}}^{\prime}=\frac{C_{1}}{r^{2}}
$$

since, at infinite distance, the lateral effective stress is $\sigma_{\mathrm{h}}^{\prime}$. Here $C_{1}$ is a constant, to be determined by the condition at any time $t$ that, when $r=r_{0}, \sigma_{r}^{\prime}=\sigma_{R 0}^{\prime}$, so that

$$
\sigma_{R 0}^{\prime}-\sigma_{\mathrm{h}}^{\prime}=\frac{C_{1}}{r_{0}^{2}}
$$

and, thus

$$
C_{1}=r_{0}^{2}\left(\sigma_{R 0}^{\prime}-\sigma_{\mathrm{h}}^{\prime}\right)
$$

and in particular, substituting equation (28) in equation (26) at $r=R$, the shock front

$$
\sigma_{R}^{\prime}-\sigma_{\mathrm{h}}^{\prime}=\frac{r_{0}^{2}\left(\sigma_{R 0}^{\prime}-\sigma_{\mathrm{h}}^{\prime}\right)}{R^{2}}
$$

But from the earlier argument, the excess pore pressure, $u_{\mathrm{p}}$, is given by

$$
u_{\mathrm{p}}=\sigma_{R}^{\prime}-\sigma_{\mathrm{p}}^{\prime}
$$

and thus

$$
u_{\mathrm{p}}=\frac{r_{0}^{2}\left(\sigma_{R 0}^{\prime}-\sigma_{\mathrm{h}}^{\prime}\right)}{R^{2}}-\left(\sigma_{\mathrm{p}}^{\prime}-\sigma_{\mathrm{h}}^{\prime}\right)
$$

The same consideration of conservation of mass applies here as in the case of external pressure on a cylinder; namely, the clay solid which occupied the volume between $R_{0}$ and $R$ at void ratio $e_{1}$ is equal to that filling the volume between $r_{0}$ and $R$ 
at void ratio $e_{2}$ after collapse. This gives a relation, as before, among $r_{0}, R$, and $R_{0}$. Ratio of new volume to old volume equals

$$
\frac{R^{2}-r_{0}^{2}}{R^{2}-R_{0}^{2}}=\frac{1+e_{2}}{1+e_{1}}=b
$$

From equation (31) an expression can be obtained for $r_{0}{ }^{2}$ for substitution in equation (30), to give

$$
u_{\mathrm{p}}=\left\{(1-b)+b \frac{R_{0}^{2}}{R^{2}}\right\}\left(\sigma_{R 0}^{\prime}-\sigma_{\mathrm{h}}^{\prime}\right)-\left(\sigma_{\mathrm{p}}^{\prime}-\sigma_{\mathrm{h}}^{\prime}\right)
$$

At the limiting value of $R$, equal to $R_{\mathrm{f}}, u_{\mathrm{p}}=0$ so that, from equation (25)

$$
(1-b)+b \frac{R_{0}{ }^{2}}{R_{\mathrm{f}}{ }^{2}}=\frac{\sigma_{\mathrm{p}}^{\prime}-\sigma_{\mathrm{h}}^{\prime}}{\sigma_{R 0}^{\prime}-\sigma_{\mathrm{h}}^{\prime}}
$$

Since $\sigma_{\mathrm{h}}^{\prime}$ is a reference stress, new terms will be defined for convenience as follows

$$
\begin{aligned}
\sigma_{R 0}^{*} & =\sigma_{R 0}^{\prime}-\sigma_{\mathrm{h}}^{\prime} \\
\sigma_{p}^{*} & =\sigma_{\mathrm{p}}^{\prime}-\sigma_{\mathrm{h}}^{\prime}
\end{aligned}
$$

in which both $\sigma_{R 0}^{*}$ and $\sigma_{\mathrm{p}}^{*}$ are still effective stresses. Thus equation (32) can be written

$$
u_{\mathrm{p}}=\left\{(1-b)+b \frac{R_{0}^{2}}{R^{2}}\right\} \sigma_{R 0}^{*}-\sigma_{\mathrm{p}}^{*}
$$

For this problem the control equation obtained from the continuity of flow out of the phase interface, and permeability-controlled flow to the inner cylindrical surface is the same as equation (7) except that $R$ is increasing so the first term is positive, and flow is taking place from the outer radius $R$ to the inner radius $r_{0}$. The equation is therefore

$$
\Delta v \frac{\mathrm{d} R}{\mathrm{~d} t} R-\frac{k_{\mathrm{s}} u_{\mathrm{p}}}{\gamma_{\mathrm{w}} \ln R / r_{0}}=0
$$

As has been seen, $u_{\mathrm{p}}$ is no longer constant, and must be replaced with equation (35). In addition, $r_{0}$ is obtained from equation (31) in terms of $b, R$ and $R_{0}$. With these substitutions, the revised form of $R^{\prime}$ from equation (9), and some rearrangement, equation (36) can be written in the form

$$
\frac{R^{3} \ln \left\{\frac{R}{\left[R^{2}(1-b)+b\right]^{1 / 2}}\right\} \mathrm{d} R}{b \sigma_{R 0}^{*}-\left[\sigma_{\mathrm{p}}^{*}-(1-b) \sigma_{R 0}^{*}\right] R^{2}}=\frac{k_{\mathrm{s}} \mathrm{d} t}{\Delta v \gamma_{\mathrm{w}} R_{0}{ }^{2}}
$$

in which, for simplicity of expression, the prime has again been dropped from $R^{\prime}$ everywhere. It is convenient to substitute for the constant terms in the denominator

$$
\left.\begin{array}{l}
A=\sigma_{\mathrm{p}}^{*}-(1-b) \sigma_{R 0}^{*} \\
B=b \sigma_{R 0}^{*}
\end{array}\right\}
$$

The solution requires integration of the left side from $R=1$ to $R$, and the right side from $t=0$ to $t$. The integration of the left side leads to a complicated solution

$$
\begin{aligned}
& \frac{(1-b)}{4}\left[\frac { u _ { \mathrm { p } } } { A } \left(\left\{R^{2}+\frac{b}{1-b}\right\} \ln \left\{R^{2}+\frac{b}{1-b}\right\}\right.\right. \\
& -R^{2} \ln R^{2}+\left(R^{2}-1\right) \ln (1-b) \\
& \left.-\left\{1+\frac{b}{1-b}\right\} \ln \left\{1+\frac{b}{1-b}\right\}\right)+\frac{u_{\mathrm{p}} B}{A^{2}} \\
& \times\left(\ln \left\{\frac{B-A}{B-A R^{2}}\right\} \ln \left\{\frac{B}{B-b(B-A)}\right\}\right. \\
& -\operatorname{Li}_{2}\left\{1-\frac{A}{B}\right\}+\operatorname{Li}_{2}\left\{1-\frac{A R^{2}}{B}\right\} \\
& +\operatorname{Li}_{2}\left\{\frac{(1-b)(B-A)}{B-b(B-A)}\right\} \\
& \left.\left.-\operatorname{Li}_{2}\left\{\frac{(1-b)\left(B-A R^{2}\right)}{B-b(B-A)}\right\}\right)\right]=\frac{u_{\mathrm{p}} k_{\mathrm{s}} t}{\gamma_{\mathrm{w}} R_{0}{ }^{2}}=T
\end{aligned}
$$

in which $u_{\mathrm{p}}$ is the initial pore pressure generated by the excess of the applied stress at the hole boundary over the maximum past effective stress, and the notation $\mathrm{Li}_{2}$ stands for the dilogarithmic function of Euler. There are a variety of notations for this function, which is apparently infrequently encountered; the notation of Lewin (1958) has been adopted here. The function is tabulated in Lewin (1958) and a closely related function in Abramowitz \& Stegun (1965), but for convenience a brief table of values is included as Appendix 1 herewith. In practical problems in soil mechanics, the arguments of the function are always less than unity. If the analytical solution, equation (39), is inconvenient, the left side of equation (39) can always be evaluated numerically from 1 to the selected value of $R$.

It can be seen that the integrand of the function on the left side of equation (37) goes to infinity when

$$
R^{2}=R_{\mathrm{f}}^{2}=B / A
$$

and the integral also becomes infinite when $R=$ $R_{\mathrm{f}}$ is used as the upper limit. This is the same value of $R_{\mathrm{f}}$ obtained from equation (33) and is the 
asymptotic value, which, as the integration indicates, is reached in infinite time.

Equation (39) is dimensionless, since $A$ and $B$ both have the dimensions of stress, and in particular the right-hand side is a dimensionless time $T$. A general graphic solution can no longer be given, but a solution from equation (39) can be plotted for specific values of the various constants. For the sake of illustration, Fig. 7 shows $R$ and $r_{0}$ plotted against the square root of dimensionless time from equation (39) for values of the constants represented by the Olga B test case. For that soil (Scott, 1989) $e_{1}=2.75, e_{2}=1.79$, and thus $b=0.744 ; \sigma_{\mathrm{p}}^{\prime}=58 \mathrm{kN} / \mathrm{m}^{2} ; \sigma_{\mathrm{h}}^{\prime}$ and $\sigma_{R 0}^{\prime}$ are chosen to be $20 \mathrm{kN} / \mathrm{m}^{2}$ and $120 \mathrm{kN} / \mathrm{m}^{2}$ respectively, so that $A=12 \cdot 4$, and $B=74 \cdot 4$. From equation (40) the dimensionless limiting radius $R_{\mathrm{f}}$ here is $2 \cdot 45$. From the dimensionless form of equation (31), where $R_{0}=1$, the limiting dimensionless value of cavity expansion, $r_{\text {of }}$, is found to equal $1 \cdot 51$.

Because of the diminution of the generating pore pressure at $R$ with the inverse square of distance, the solution to the internal cavity problem no longer exhibits the square root of time dependency at small times shown by the external loading cases. The solution also approaches the limiting radius $R_{\mathrm{f}}$ much more slowly than previously, and, indeed, requires infinite time to reach it. In this case, the progress of the solution can also be represented by modified forms of equations (20) and (24)

$$
\begin{aligned}
& \text { dimensionless displacement, } U_{\mathrm{r}}=\frac{r_{0}-1}{r_{\mathrm{Of}}-1} \\
& \text { volumetric consolidation, } U_{\mathrm{rv}}=\frac{R^{2}-1}{R_{\mathrm{f}}{ }^{2}-1}
\end{aligned}
$$

and these solutions are shown in Fig. 8. For the same data, the one-dimensional plane solution

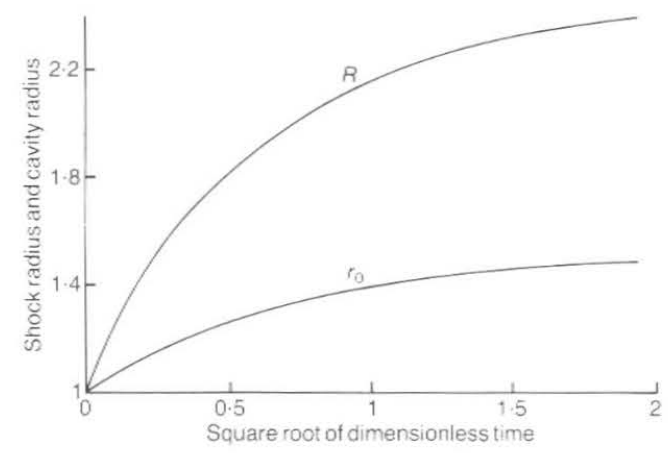

Fig. 7. Solutions for movement of phase-change surface as a function of dimensionless time for circular cylindrical cavity expansion case; constants for Olga site sensitive clay (Mesri \& Choi, 1985) as given in text
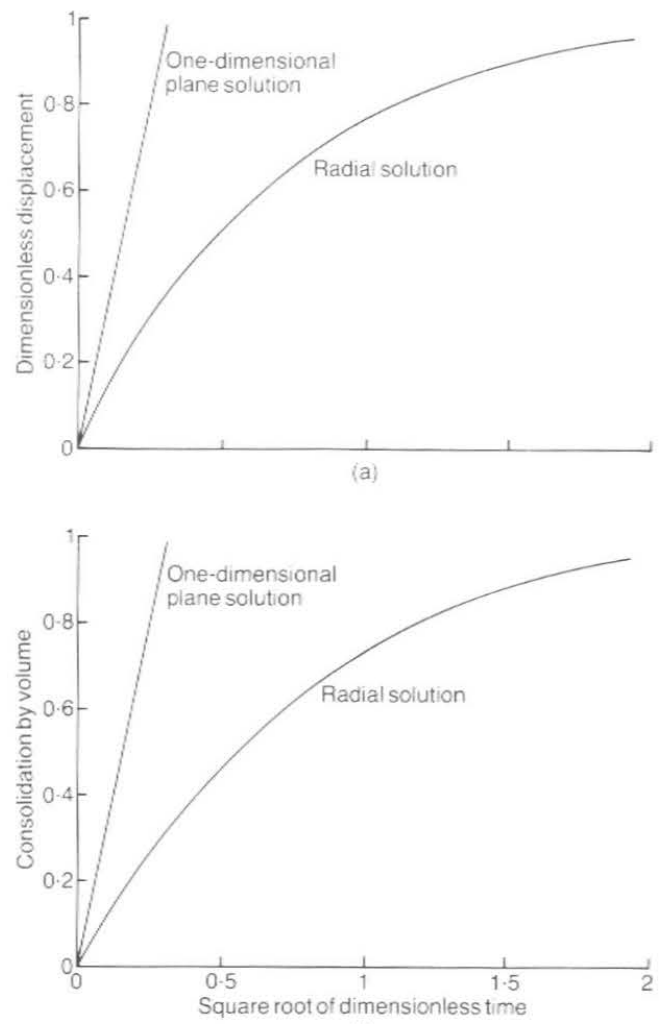

(b)

Fig. 8. Solutions for consolidation for cylindrical cavity expansion case, Olga site: (a) dimensionless displacement $U_{\mathrm{r}}=\left(r_{0}-1\right) /\left(r_{01}-1\right)$ plotted against $T^{1 / 2}$; (b) volumetric consolidation $U_{\mathrm{rv}}=\left(R^{2}-1\right) /\left(R_{\mathrm{f}}^{2}-1\right)$ plotted against $T^{1 / 2}$

(Scott, 1989) is also shown. At different times in the progress of the problem, the excess pore pressures will appear as the dashed lines in Fig. 6, beginning at the displaced radii $r_{01}, r_{02}$ and so on, to reach zero at all radii when $r_{\mathrm{Of}}$ is reached.

Although, in this example, for comparison with the previous solution (Scott, 1989) the values of the maximum past vertical effective stress have been used, the reference stress to be employed in the case of a test in a real vertical borehole into a sensitive clay will be the maximum past lateral effective stress. The solution of the problem of the expansion of a spherical cavity in an infinite clay medium can also be accomplished, but would seem to have little value in view of the strong anisotropy present in an overconsolidated sensitive clay.

Three constants are evident in the dimensionless form of the solution of the radial cavity expansion problem, $A, B$, and $b$, and they in turn involve the stress $\sigma_{R 0}^{\prime}$, the applied pressure, $\sigma_{\mathrm{p}}^{\prime}$ (the maximum past effective stress) and $\sigma_{\mathrm{h}}^{\prime}$ (the 
existing horizontal effective stress in the soil), as well as the before and after collapse void ratios $e_{1}$ and $e_{2}$. Complete evaluation of a test also requires knowledge of the post-collapse permeability of the clay.

\section{CONCLUSION}

Solutions have been presented for several cases of consolidation of a sensitive clay exhibiting phase change or collapse behaviour at applied stresses above the previous maximum past effective stress. The problems include radial consolidation due to either external stressing of an infinite clay cylinder or internal stressing of a cylindrical cavity in an infinite clay mass, and spherical consolidation of a sphere under external loading. Applications include consolidation of triaxial specimens of appropriate clay in the laboratory, and a field test of the pressure-meter type, which will, however, require modification, to permit drainage at the cavity boundary.

All the solutions given are capable of extension to the 'more complicated' model described previously (Scott, 1989).

\section{APPENDIX 1. DILOGARITHMIC FUNCTION}

\begin{tabular}{|c|c|c|c|c|c|}
\hline$x$ & $\mathrm{Li}_{2}(x)$ & $x$ & $\mathrm{Li}_{2}(x)$ & $x$ & $\mathrm{Li}_{2}(x)$ \\
\hline 0.00 & 0.000000 & $0 \cdot 34$ & 0.374341 & 0.68 & 0.855427 \\
\hline 0.01 & 0.010025 & $0 \cdot 35$ & $0 \cdot 386605$ & 0.69 & 0.872291 \\
\hline 0.02 & 0.020100 & $0 \cdot 36$ & 0.398958 & $0 \cdot 70$ & 0.889377 \\
\hline .03 & 0.030228 & $0 \cdot 37$ & 0.411400 & 0.71 & 0.906694 \\
\hline .04 & 0.040407 & $0 \cdot 38$ & 0.423933 & 0.72 & 0.924250 \\
\hline 0.05 & 0.050639 & $0 \cdot 39$ & 0.436560 & 0.73 & 0.942057 \\
\hline .06 & 0.060924 & 0.40 & 0.449282 & 0.74 & 0.960126 \\
\hline .07 & 0.071264 & 0.41 & 0.462102 & 0.75 & 0.978469 \\
\hline 0.08 & 0.081659 & 0.42 & 0.475021 & 0.76 & 0.997099 \\
\hline 0.09 & 0.092110 & 0.43 & 0.488 & 0.77 & 016030 \\
\hline $0 \cdot 10$ & & 0.44 & 0.50 & 0.78 & \\
\hline $0 \cdot 11$ & 0.113182 & 0.45 & & 0.79 & \\
\hline $0 \cdot 12$ & $0 \cdot 123806$ & 0.46 & 0.527 & 0.80 & 1.074794 \\
\hline$\cdot 13$ & 0.134 & 0.47 & 0.54 & 0.81 & \\
\hline $0 \cdot 14$ & 0.145231 & 0.48 & 0.55 & 0.82 & 5808 \\
\hline$\cdot 15$ & $0 \cdot 156 C$ & 0.4 & & 0.83 & 6936 \\
\hline 16 & $0 \cdot 16$ & $0 \cdot 50$ & 0.58 & 0.84 & 58516 \\
\hline 0.17 & 0.177829 & $0 \cdot 51$ & 0.596165 & 0.85 & $1 \cdot 180580$ \\
\hline $0 \cdot 18$ & 0.1888 & 0.5 & & 0.8 & \\
\hline 0.19 & 0.199879 & 0.53 & 0.624 & 0.87 & \\
\hline $0 \cdot 20$ & 0.211003 & 0.54 & 0.638708 & 0.88 & 1.250083 \\
\hline $0 \cdot 21$ & 0.2221 & 0.5 & & 0.8 & \\
\hline 0.22 & 0.233453 & 0.56 & 0.667 & 0.90 & $1 \cdot 299701$ \\
\hline 0.23 & 0.244782 & 0.57 & 0.682479 & 0.91 & 1.325702 \\
\hline $0 \cdot 24$ & 0.256181 & 0.58 & 0.697361 & 0.92 & \\
\hline 0.25 & 0.267652 & 0.59 & 0.712 & 0.93 & $1 \cdot 380590$ \\
\hline $0 \cdot 26$ & 0.279196 & 0.60 & 0.727586 & 0.94 & 1.409747 \\
\hline $0 \cdot 27$ & 0.290815 & 0.61 & 0.742939 & 0.95 & 1.440283 \\
\hline $0 \cdot 28$ & 0.302 & 0.6 & 0.758460 & 0.9 & 1.472439 \\
\hline 0.29 & 0.314280 & 0.63 & 0.774153 & 0.97 & 1.506525 \\
\hline $0 \cdot 30$ & 0.326129 & 0.64 & 0.790026 & 0.9 & 1.542946 \\
\hline 0.31 & & 0.6 & & 0.9 & \\
\hline 0.32 & 0.350069 & 0.66 & 0.822330 & 1.00 & 1.625132 \\
\hline 0.33 & 0.362163 & 0.67 & 0.838776 & & \\
\hline
\end{tabular}

\section{REFERENCES}

Abramowitz, M. \& Stegun, I. A. (eds) (1965). Handbook of mathematical functions. New York: Dover.

Cryer, C. W. (1963). A comparison of the threedimensional consolidation theories of Biot and Terzaghi. Quart. J. Mech. Appl. Math. 16, 401-412.

de Leeuw, E. H. (1964). Consolidatie in drie dimensies. Delft Lab. Grondmech. Med. 9, 17-46.

Lewin, L. (1958). Dilogarithms and associated functions. London: Macdonald.

Mesri, G. \& Choi, Y. K. (1985). Settlement analysis of embankments on soft clays. J. Geotech. Engng, Am. Soc. Civ. Engrs 111, No. 4, 441-464.

Mesri, G. \& Rokhsar, A. (1983). Theory of consolidation for clays. J. Geotech. Engng, Am. Soc. Civ. Engrs 109, No. 6, 873-878.

Scott, R. F. (1963). Principles of soil mechanics. Reading, Massachusetts: Addison-Wesley.

Scott, R. F. (1989). Consolidation of sensitive clay as a phase change process. J. Geotech. Engng, Am. Soc. Civ. Engrs 115, Oct., No. 10, 1439-1458. 